\title{
Article
}

\section{Post discharge medicines use review (dMUR) service for older patients: Cost- savings from community pharmacist interventions}

Ramsbottom, Helen, Rutter, Paul and Fitzpatrick, Ray

Available at http://clok.uclan.ac.uk/17253/

Ramsbottom, Helen, Rutter, Paul ORCID: 0000-0003-4106-1515 and

Fitzpatrick, Ray (2018) Post discharge medicines use review (dMUR) service for older patients: Cost-savings from community pharmacist interventions. Research in Social and Administrative Pharmacy, 14 (2). pp. 203-206. ISSN 1551-7411

It is advisable to refer to the publisher's version if you intend to cite from the work. http://dx.doi.org/10.1016/j.sapharm.2017.02.007

For more information about UCLan's research in this area go to http://www.uclan.ac.uk/researchgroups/ and search for <name of research Group>.

For information about Research generally at UCLan please go to http://www.uclan.ac.uk/research/

All outputs in CLoK are protected by Intellectual Property Rights law, including Copyright law. Copyright, IPR and Moral Rights for the works on this site are retained by the individual authors and/or other copyright owners. Terms and conditions for use of this material are defined in the policies page. 


\title{
Post discharge medicines use review (dMUR) service for older patients: Cost-savings from community pharmacist interventions
}

\author{
Helen Ramsbottom a, b, *, Paul Rutter a , Ray Fitzpatrick ${ }^{\mathrm{c}}$ \\ a School of Pharmacy and Biomedical Sciences, Maudland Building, University of Central Lancashire, Preston, PR1 2HE, UK \\ b Southport and Ormskirk Hospital NHS Trust, Town Lane, Southport, PR8 6PN, UK \\ ${ }^{\text {c }}$ School of Pharmacy, Keele University, Staffordshire, ST5 5BG, UK
}

\section{A R T I C L E I N F O}

\section{Article history:}

Received 10 February 2017

Accepted 10 February 2017

\section{Introduction}

The likelihood that an older medical patient will be discharged from hospital on the same medications upon which they were admitted is less than $10 \%{ }^{1}$ with incomplete documentation of changes in medication linked to preventable readmissions. ${ }^{2,3}$

Improved discharge information and patient education are core principles for reducing unintentional medication changes, patient harm and hospital re-admissions. ${ }^{4,5}$ In the United Kingdom (UK), Medicines Use Reviews (MURs), face-to-face consultations between a patient and their regular community pharmacist, are designed to identify any problems or information needs patients have with medicines and offer solutions. ${ }^{6}$ This service is free to National Health Service (NHS) patients; pharmacists receive a fee (currently $£ 28$ ) per MUR provided. Since 2011, the MUR service has targeted specific patient groups, identified as being at particular risk of medication related problems or poor adherence, including patients recently discharged from hospital. ${ }^{6}$ Early evidence suggests uptake of post discharge MURs (dMURs) has been poor. ${ }^{7,8}$

In 2012, a Department of Health steering group recommended that formal communication channels between hospital and community pharmacy should be established so that dMURs become an integral part of the medicines pathway. ${ }^{4}$ In light of the documented medication problems that occur on transfer of care, a randomized controlled feasibility study (the 'Medicines Support Study') was devised to explore the effect a hospital referral system to older

\footnotetext{
* Corresponding author. Pharmacy Department, Southport and Ormskirk NHS Trust, Town Lane, Kew, Southport, PR8 6PN, UK.

E-mail address: helen.ramsbottom@nhs.net (H. Ramsbottom).
}

patients' regular community pharmacists had on their care. Findings regarding problems in patient recruitment to the study and community pharmacists' perceptions on delivering dMURs have already been reported. ${ }^{9,10}$ This paper reports on the potential clinical and economic impact of interventions made by the pharmacists.

\section{Methods}

All pharmacists working on medical wards at Southport and Ormskirk Hospitals NHS Trust, England identified in-patients aged over 65 years who, in their professional opinion, could benefit from a dMUR. Patients were given written information about the study before being visited by the researcher (HR) who assessed eligibility for recruitment. Inclusion and exclusion criteria have previously been reported. ${ }^{9}$ Ethics approval for the study was obtained from the Northwest Research Ethics Committee (Ref 13/NW/0779).

Consenting patients were randomized to receive either a dMUR or standard discharge care. A referral form and discharge prescription for those to receive a dMUR was faxed to their nominated community pharmacist to allow completion within 28 days as per the national service specification. Participating community pharmacists were asked to share the 'action plan' on completion of each dMUR with HR. This action plan included all advice given to the patient and recommendations made to the patient's family doctor. Each piece of advice given or recommendation made was classed as an intervention for the purposes of this study. Basic descriptive data analysis on the numbers of dMURs completed and the manner in which they were performed were conducted.

To assess the potential clinical impact of interventions made an expert panel was convened and Delphi methodology used to arrive at consensus. The term "expert" in this context has been described as "clinicians practicing in the field under consideration". ${ }^{11}$ Therefore the expert panel for this study comprised 5 pharmacists, 2 medical practitioners based in academia and 2 family doctors.

Consensus was defined as 75\% agreement between participants' scores as advocated by Diamond et al. ${ }^{12}$ In addition, consensus can also be achieved when stability of the distribution of scores occurs. $^{13,14}$ If variation between rounds is less than $15 \%$, a state of 
stability is said to exist. ${ }^{12,14}$ It was decided to use $75 \%$ agreement as the primary target, but also to consider the variance in scores if the $75 \%$ threshold was not reached.

All recorded interventions were collated and sent to panel members via email to score independently. Participants were instructed to award each intervention a value according to the Eadon scoring criteria (Table 1 ). ${ }^{15}$ Space for free text comments was provided after each statement, allowing participants to provide a rationale for their positions.

Participants were asked to return their scores within two weeks. Reminder emails were sent at 2 and 4 weeks if no reply had been received. If no response was obtained by week 6 , that participant was viewed as having left the process.

Following the return of scores, HR collated all scores and freetext comments. Each participant then received a personalised 'Round Two' survey, with box plots showing their score along with median and upper and lower quartiles for group scores. These personalized surveys were then circulated to participants, returned and analysed using the same process as for 'Round One'.

Once consensus was achieved, the School of Health and Related Research (ScHARR) model, used to apply an 'opportunity cost' saving to medication errors and adverse drug events avoided, was mapped onto the Eadon score to determine the economic impact of pharmacist interventions. ${ }^{16,17}$

\section{Results}

During the 9-month study, 30 patients were referred to their regular community pharmacist, resulting in 20 dMURs conducted (67\% completion rate). Twelve (60\%) were completed as per study protocol (defined as dMUR completion within 4 weeks of discharge). The others were completed after 4 weeks, following one or more prompts from HR.

Patients being unable to visit the pharmacy or being noncontactable were the main reasons why dMURs were not completed as per protocol, although some pharmacists reported not receiving the original referral or this had been lost by the pharmacy.

Action plans were returned for 17 of the 20 dMURs, generating 35 interventions. The most common type of intervention $(n=14$, $40 \%$ ) was provision of information to improve their understanding of why and how they were taking their medicines; the medical condition for which the medicines were being used; how to avoid side effects, or a combination. Six interventions (17\%) involved medicines reconciliation to ensure discrepancies between preadmission and discharge medications were intentional and understood. Four interventions resulted in the provision or recommendation of a medication compliance aid (such as a monitored dosage system or a spacer device to aid inhaler technique). A further 4 involved patient referral for monitoring of their medication/condition. Three interventions involved provision of lifestyle advice, for example regarding smoking cessation. The remaining 4 interventions included advice on how to obtain further supplies of medication, referral to the family doctor for consideration of possible additional medication, advice on over-the-counter medication and referral to a specialist diabetes nurse.

\subsection{Impact of pharmacist intervention}

Five of the 35 reported interventions were very similar in nature and for the purpose of the Delphi review were considered to be the same intervention; hence 30 interventions in total were reviewed by the panel.

Eight participants (all five pharmacists, both academic clinicians and one of the two family doctors) returned Round One surveys, and seven participants (all five pharmacists, one academic clinician and one family doctor) returned Round Two surveys. Following Round Two, greater than 75\% agreement in scores was achieved for 5 of the 30 interventions, although 8 more achieved 71\% agreement. A further 8 achieved $57 \%$ agreement. When the percentage change in mean scores between Rounds One and Two were calculated, the variation was less than $10 \%$ for 28 of the 30 interventions, and less than $15 \%$ for all but one. This indicated that participants were not likely to alter their scores very much if a third round was undertaken and at the risk of further drop-outs a further round was not conducted.

None of the interventions made by community pharmacists were found to be detrimental to the patient's wellbeing. Fifteen interventions (50\%) were assessed as being significant and leading to an improvement in patient care, whilst one intervention, where a prescription for pre-admission medication was identified and referred back to the doctor by the community pharmacist, was graded as highly significant and preventing major organ failure or adverse reaction of similar importance (Table 2). This patient had been diagnosed with heart failure during admission and had had several changes to medicines including diuretics, anticoagulants, beta-blockers and angiotensin converting enzyme inhibitors.

\section{Discussion}

In this feasibility study, two-thirds of participants received a dMUR; more than twice that reported in a recent study of an electronic referral (e-referral) system in Northeast England. ${ }^{18}$ The higher completion rate in the current study may be because the researcher contacted all community pharmacists at 4 weeks post hospital referral to prompt completion of the dMUR. Such an approach, whilst feasible in this study, is unlikely to be sustainable on scale-up when accounting for secondary care pharmacy staff workloads. ${ }^{9}$ However, the ability to build a 'prompt' into an ereferral system may be a workable solution to help increase completion rates.

In this study, the most common reason for non-completion at 4 weeks was patients' inability to visit the pharmacy, affecting almost a quarter of participants. This was unexpected, as it was one of the study exclusion criteria, and indicates that despite trying to screen out this type of patient at recruitment, poor mobility affecting the elderly post-discharge population is a major barrier to dMURs being conducted. The current national MUR service specification and

Table 1

Eadon scoring system.

\begin{tabular}{ll}
\hline Intervention type & Score \\
\hline Intervention which is detrimental to the patients well-being & 1 \\
Intervention is of no significance to patient care & 2 \\
Intervention is significant but does not lead to an improvement in patient care & 3 \\
Intervention is significant and results in an improvement in the standard of care & 4 \\
Intervention is very significant and prevents a major organ failure or adverse reaction of similar importance & 5 \\
Intervention is potentially life-saving & 6 \\
\hline
\end{tabular}


Table 2

Eadon Scores and ScHARR cost avoidance associated with pharmacist interventions.

\begin{tabular}{|c|c|c|c|}
\hline Eadon score & ScHARR cost avoidance $(£)$ & Number of interventions & Total cost avoidance $(£)$ \\
\hline $2-3$ & $0-6$ & 14 & $0-84$ \\
\hline 4 & $65-150$ & 15 & $975-2250$ \\
\hline 5 & $713-1484$ & 1 & $713-1484$ \\
\hline 6 & $1085-2120$ & 0 & - \\
\hline \multirow{2}{*}{\multicolumn{3}{|c|}{$\begin{array}{l}\text { Overall Total Cost Avoidance } \\
\text { Cost Avoidance per dMUR }\end{array}$}} & $£ 1688-£ 3818$ \\
\hline & & & $£ 112.53-£ 254.53$ \\
\hline
\end{tabular}

${ }^{\mathrm{a}}$ Mean $=2$ interventions per dMUR.

community pharmacy contract make it difficult for pharmacists to undertake MURs at patients' homes, so modifications are needed if dMURs are to be routinely offered to this population.

Patients also failed to receive a dMUR as they were not contactable by the community pharmacy. The reasons for this are unclear, although within the study around one fifth of patients were re-admitted to hospital within four weeks of discharge, and through participant interviews [not reported here], it was apparent that patients had multiple appointments as part of their care package post discharge. It is therefore possible that patients may not have been at home at the times community pharmacists tried to contact them. This does raise questions over co-ordination of care by the various healthcare agencies involved in supporting elderly patients discharged from hospital and the need for better communication.

For those dMURs completed, an average of 2 interventions per dMUR were instigated. Most commonly, interventions involved the provision of information to improve patient understanding of their medication and how to use it in the most effective, convenient and safe way. This type of medicine support is in line with the ethos of the MUR service as a whole. However, nearly one in five cases required intervention to reconcile discrepancies between preadmission and discharge medications. These type of interventions exemplify the ease with which misunderstandings can arise when changes to medication occur during care transitions. Such misunderstandings could result in inappropriate medicine taking and further demonstrate the risks to patients during transfer of care. ${ }^{19}$

Following expert review, almost $60 \%$ of interventions were deemed to improve clinical care. This is similar to the 53\% reported in Eadon's original (hospital-based) study, but lower than the $84 \%$ reported during an analysis of the effect of consultant pharmacist case management of older people in intermediate care. ${ }^{15,17}$ This lower value is perhaps expected as, community pharmacists are unlikely to possess the resources (in terms of specialist knowledge, multidisciplinary team support, time for repeated follow-up and ability to conduct home visits) afforded to a consultant pharmacist in care of the elderly.

Assigning a monetary value to these interventions appears to show a four-fold return on investment, even when using the most conservative estimate of value delivered per dMUR conducted. This figure does not however factor in hospital pharmacist time costs associated with patient recruitment, referral and follow-up. The staff costs in this study approximated to $£ 42.44$ for each referral that resulted in a dMUR. After adding the MUR fee, the final cost of the service would be $£ 70.44$ per completed dMUR. This still represents a substantial cost saving to the NHS. Further cost savings could be made if referrals were managed by pharmacy support staff. $^{18}$

The small number of patients in this study means findings need to be interpreted with caution. Additionally, interventions studied were not outcomes in themselves, and their conversion into cost savings is an estimation, as it is difficult to demonstrate a direct effect of the interventions made. However, the use of the Eadon-
ScHARR model to assign clinical significance and monetary value to each intervention provides an insight into the effect of these interventions on patient care. It should also be noted that using a Delphi process to gain consensus is still based on subjective opinion. ${ }^{13}$ This is particularly pertinent here as, consensus regarding the score to award the majority of interventions was defined by stability in the opinions of the reviewers, not agreement as to the score awarded.

A larger study involving more interventions would help to validate these results, and if potential clinical significance and cost avoidance could be linked to other outcomes such as reduced readmission rates, this would help to demonstrate causality of the intervention on improvements in these outcomes.

\section{Conclusion}

This study shows the potential for improved patient care and a positive return on investment when community pharmacists perform dMURs. However, operational barriers exist to delivering dMURs to the frail elderly and there is a need to improve communication channels between health care practitioners to deliver a holistic package of care post discharge.

\section{Funding}

This research did not receive any specific grant from funding agencies in the public, commercial, or not-for-profit sectors.

\section{Conflict of interests}

None to declare.

\section{References}

1. Mansur N, Weiss A, Beloosesky Y. Relationship of in-hospital medication modifications of elderly patients to postdischarge medications, adherence, and mortality. Ann Pharmacother. 2008;42:783-789.

2. Witherington EMA, Pirzada OM, Avery AJ. Communication gaps and readmissions to hospital for patients aged 75 years and older: observational study. Qual Saf Health Care. 2008;17:71-75.

3. Davies EC, Green CF, Mottram DR, Rowe PH, Pirmohamed M. Emergency readmissions to hospital due to adverse drug reactions within 1 year of the index admission. Br J Clin Pharmacol. 2010;70:749-755.

4. Steering Group on Improving the Use of Medicines (for better outcomes and reduced waste). Improving the Use of Medicines for Better Outcomes and Reduced Waste: An Action Plan. Department of Health; 2012. Available at https://www. gov.uk/government/publications/action-plan-for-improving-the-use-ofmedicines-and-reducing-waste.

5. Royal Pharmaceutical Society (RPS). Keeping Patients Safe when They Transfer between Care Providers - Getting the Medicines Right. Final Report. RPS; 2012. Available at http://www.rpharms.com/current-campaigns-pdfs/rps-transferof-care-final-report.pdf.

6. Pharmaceutical Services Negotiating Committee (PSNC) and NHS Employers. Medicines Use Review and Prescription Intervention Service Specification. PSNC and NHS Employers; 2013. Available at http://psnc.org.uk/wp-content/ uploads/2013/06/MUR-service-spec-Aug-2013-changes_FINAL.pdf.

7. Royal Pharmaceutical Society. Keeping Patients Safe when They Transfer between Care Providers - Getting the Medicines Right. Individual Reports from the Early Adopter Sites. London: RPS; 2012. Available at http://www.rpharms.com/ 
current-campaigns-pdfs/eas-finalreports.pdf.

8. Royal Pharmaceutical Society (RPS) Innovator's Forum. Hospital Referral to Community Pharmacy: An Innovator's Toolkit to Support the NHS in England. London: RPS; 2014. Available at http://www.rpharms.com/support-pdfs/ 3649--rps--hospital-toolkit-brochure-web.pdf.

9. Ramsbottom H, Fitzpatrick R, Rutter P. Post discharge medicines use review (dMUR) service for older patients: recruitment issues in a feasibility study. Int J Clin Pharm. 2016;38:208-212. http://dx.doi.org/10.1007/s11096-015-0243-8.

10. Rutter P, Ramsbottom H. Community pharmacist perceptions of delivering post-hospital discharge medicines use reviews for elderly patients. Int J Clin Pharm. 2016. http://dx.doi.org/10.1007/s11096-016-0400-8.

11. Jones J, Hunter D. Consensus methods for medical and health services research. BMJ. 1995:311(7001):376-380.

12. Diamond IR, Grant RC, Feldman BM, et al. Defining consensus: a systematic review recommends methodologic criteria for reporting of Delphi studies. J Clin Epidemiol. 2014;67:401-409.

13. Grisham T. The Delphi technique: a method for testing complex and multifaceted topics. Int J Manag Proj Bus. 2009;2:112-130.

14. Linstone HA, Turoff M, eds. The Delphi Method. Techniques and Applications. Reading, MA: Addison- Wesley; 1975.

15. Eadon H. Assessing the quality of ward pharmacists' interventions. Int J Pharm Pract. 1992;1:145-147.

16. Karnon J, McIntosh A, Dean J, et al. Modelling the expected net benefits of interventions to reduce the burden of medication errors. J Health Serv Res Pol. 2008; 13:85-91.

17. Miller EFR, Darcy CM, Friel ABM, Scott MG, Toner SB. Consultant pharmacist case management of older people in intermediate care: a new innovative model. Eur J Pers Center Healthc. 2016;4(1):46-52.

18. Nazar $\mathrm{H}$, Brice $\mathrm{S}$, Akhter $\mathrm{N}$, et al. New transfer if care initiative of electronic referral from hospital to community pharmacy in England: a formative service evaluation. BMJ Open. 2016;6:e012532. http://dx.doi.org/10.1136/bmjopen2916-012532.

19. Coleman EA, Smith JD, Raha D, Min S. Posthospital medication discrepancies: prevalence and contributing factors. Arch Intern Med. 2005;165:1842-1847. 